\title{
FOXO3 Inhibits Human Gastric Adenocarcinoma (AGS) Cell Growth by Promoting Autophagy in an Acidic Microenvironment
}

\author{
Yuan Gao $\quad$ Weiwei Qib Libin Sun ${ }^{b} \quad$ Jing Lv ${ }^{b} \quad$ Wensheng Qiu ${ }^{b} \quad$ Shihai Liuc \\ aQingdao University, Qingdao, Shandong, ${ }^{\mathrm{b}}$ Department of Oncology, Affiliated Hospital of Qingdao \\ University, Qingdao, Shandong, 'Central Laboratory, Affiliated Hospital of Qingdao University School, \\ Qingdao, Shandong, China
}

\section{Key Words \\ Foxo3 - Autophagy $•$ Acidic microenvironment $•$ Gastric tumour}

\begin{abstract}
Background/Aims: Previous studies have shown that FOXO3, a member of the forkhead box O (FOXO) family, regulates autophagy in various cells. To date, whether the induction of autophagy in gastric cancer (GC) cells is triggered by an acidic microenvironment is unclear. Little is known about the relationship between $\mathrm{FOXO} 3$ and acidic microenvironments in GC. The aims of our study were to investigate the roles of $\mathrm{FOXO} 3$ and the acidic microenvironment in autophagy and to determine how FOXO3 and the acidic microenvironment regulate GC cell growth through autophagy. Methods: We cultured human gastric adenocarcinoma (AGS) cells in media with different $\mathrm{pH}$ values in vitro, transfected the cells with $\mathrm{FOXO} 3 a$ plasmids and then detected autophagy in the cells under different conditions. In addition, we also performed cell counting kit 8 (CCK8), wound and cell invasion assays to test cell viability and invasion, respectively. We employed real-time PCR, western blotting and mRFP-GFP-LC3 vectors to detect the expression of various autophagy indicators. Results: We found that cells treated with FOXO3 and exposed to an acidic microenvironment displayed suppressed growth compared with control cells. We also found that the protein expression levels of several autophagy makers, such as LC3I, LC3II and Beclin-1, were higher in FOXO3 plasmidtransfected AGS cells cultured in an acidic microenvironment than in control cells, while P62 protein expression levels were clearly decreased in FOXO3 plasmid-transfected cells compared with control cells. Moreover, we observed autophagic flux in AGS cells overexpressing FOXO3 and exposed to low pH conditions. Conclusion: These findings suggest that FOXO3 inhibits
\end{abstract}

Y. Gao and W. Qi contributed equally to this work.

Wensheng Qiu

and Shihai Liu

KARGER
Department of Oncology, Affiliated Hospital of Qingdao University

Qingdao, Shandong 266000 (China)

Tel.17853299199, E-Mail wsqiusci@163.com; shliumed@126.com 
AGS cell growth by promoting autophagy in an acidic microenvironment. Furthermore, the results showed that anticancer therapies targeting $\mathrm{FOXO} 3$ and low $\mathrm{pH}$ conditions may be useful in the treatment of GC.

\section{Introduction}

Gastric cancer (GC) is one of the most common types of cancer and ranks as the second leading cause of cancer-related death worldwide [1]. Currently, the most effective treatment for localized GC is surgery; however, almost half of affected patients relapse and develop advanced-stage disease after tumour removal. Moreover, although effective therapies have greatly improved five-year survival rates in patients with GC, the prognoses of patients with $\mathrm{GC}$ remain relatively poor. Patients with advanced disease are treated with chemotherapy and radiation therapy. However, the efficacy of these treatments is limited by their side effects [2]. Therefore, studies aiming to develop new anticancer agents and potent treatment strategies for patients with GC are urgently needed.

Evidence assembled over the past few years has shown that the $\mathrm{pH}$ of human tumours is, for the most part, lower than that of normal tissues [3]. Thus, tumour acidosis is an important factor in various aspects of disease progression, including growth, invasion, neoangiogenesis, and genetic instability. In addition, cancer cells develop mechanisms for surviving in acidic microenvironments. Therefore, many researchers have investigated the feasibility of regulating tumour $\mathrm{pH}$ to treat cancer [4].

The tumour microenvironment is very different from the physiologic microenvironment, as the tumour microenvironment is more acidic and is hypoxic, and nutrient deprived compared with its counterpart. These conditions can induce autophagy, thereby providing cells with an energy source with which to maintain cellular homeostasis; thus, autophagy can protect cells against acidic microenvironments [5, 6]. We propose that autophagy can be stimulated by the acidic tumour microenvironment and that the acidic tumour microenvironment and autophagy interact with each other.

Autophagy, which digests damaged cytoplasmic proteins, macromolecules and organelles, is a catabolic process through which cellular homeostasis is maintained. According to previous studies, autophagy is a self-sufficient program that improves tumour cell viability under adverse microenvironmental conditions. However, increasing evidence shows that the autophagy induced by cancer cells serves as a dynamic mechanism that either supports or inhibits the proliferation and growth of targeted tumours [7]. However, whether autophagy is involved in cell death is a controversial issue. The involvement of autophagy in cell death appears to depend on tumour types and stages and the strength of the autophagyinducing stimulus [2]. Many researchers believe that autophagy and apoptosis often occur simultaneously in cells and that autophagy often leads to apoptosis. Based on the afore mentioned findings, it may be beneficial to develop a novel anticancer agent that induces autophagy and apoptosis simultaneously. Clarifying the role of autophagy in tumour cells may be beneficial with respect to the development of new therapies.

Previous studies have shown that FOXO3, a member of the forkhead box 0 (FOXO) transcriptional protein family, is involved in regulating autophagy [8]. FOXO3 has been shown to function as a downstream effector of serine/threonine protein kinase B (PKB)/ Akt. When activated by PI3K/Akt pathway inhibition, FOXO3 modulates several cellular processes, as it induces autophagy and inhibits metastasis and apoptotic cell death [9]. FOX03 phosphorylation by Akt induces the release of the protein from the nucleus, as well as its degradation, leading to the inhibition of transcription [10]. Some evidences have showed that FOXO3 may facilitate the survival and metastasis of cancer cells. However, some evidences have showed that FOXO3 may facilitate the survival and metastasis of cancer cells [11]. But additionally, in recent years, FOXO3 has been demonstrated to activate autophagy in atrophying muscle cells, breast cancer cells and oesophageal cancer cells [12-14]. However, the role of FOXO3 in GC remains unclear. We speculate that FOXO3 inhibits human gastric 


\section{Cellular Physiology Cell Physiol Biochem 2018:49:335-348

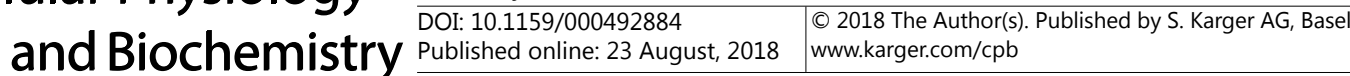

Gao et al.: FOXO3 Inhibits AGS Cell Growth via Autophagy

adenocarcinoma (AGS) cell growth by inducing autophagy.

In this study, we determined whether FOXO3 harnesses autophagy to kill GC cells by exerting proapoptotic effects in an acidic microenvironment.

\section{Materials and Methods}

\section{Cell culture}

The AGS cells were acquired from Cell Research Company and were maintained in F12K (CELL RESEARCH, $500 \mathrm{ml}$ ) supplemented with 10\% FBS (Gibco, New York, USA) containing 100 units/mL penicillin and $100 \mu \mathrm{g} / \mathrm{mL}$ streptomycin (HyClone Laboratories). The cells were maintained at $37^{\circ} \mathrm{C}$ in a humidified atmosphere of 5\% $\mathrm{CO} 2$. The medium was replaced every 2 days. The $\mathrm{pH}$ value of medium was adjusted by the addition of $1 \mathrm{M} \mathrm{HCl}$. The cells were randomly assigned to four groups incubated at $\mathrm{pHs}$ of 6.0 or 7.4. We monitored the cells by microscopy to ensure that they maintained their original morphology. The $\mathrm{pH}$ of the medium in each group was measured before every experiment to ensure that the cells were exposed to stable experimental conditions.

\section{Transfection of FOXO3 plasmids}

Plasmids expressing active Flag-tagged FOXO3 or a non-DNA-binding Flag-tagged FOXO3 (which served as a negative control) were provided by GeneChem. We divided the plasmids into the following two groups: a control group (no FOXO3) , and a positive FOXO3 expression group. Each plasmid construct was transfected into AGS cells. Endogenous FOXO3 expression was detected by real-time PCR after 24 hours.

For the transfection experiments, the cells were seeded in 24-well plates and incubated overnight at $37^{\circ} \mathrm{C}$ with $5 \% \mathrm{CO} 2$. The above plasmids were transfected into the cells using Lip3000 (Life Technologies), according to the manufacturer's protocol. The AGS cells were then cultured for $24 \mathrm{~h}$ at $37^{\circ} \mathrm{C}$ with $5 \% \mathrm{CO} 2$.

\section{CCK8 assay for cell viability}

Cell viability was detected using cell counting kit 8 (CCK) assay [15]. Cells exposed to media with different $\mathrm{pH}$ values (6.0 and 7.4) were inoculated in 96-well culture plates at a density of $4 \times 10^{3}$ per well. Each well contained $100 \mu \mathrm{L}$ of medium. The cells were incubated overnight, after which the medium was replaced with $100 \mu \mathrm{L}$ of fresh medium containing 10\% CCK8 (WST-8, yiyuanbiotech). The cells were subsequently incubated for $1-3 \mathrm{~h}$ at $37^{\circ} \mathrm{C}$ with $5 \% \mathrm{CO} 2$, and the absorbance was measured at $450 \mathrm{~nm}$ with a microplate spectrophotometer. Cell viability was calculated as follows: Cell viability (\%) $=[$ A450 (sample) - A450 (blank)/A450 (control) - A450 (blank)]×100\%.

\section{Cell wound scrape assay}

Cells from each group were seeded in 6-well plates. A small wound was introduced in the confluent monolayer with a 200- $\mu \mathrm{L}$ pipette tip. The cells were then washed with PBS and incubated in serum-free F12k medium for $24 \mathrm{~h}$ at $37^{\circ} \mathrm{C}$ with $5 \% \mathrm{CO} 2$. Thereafter, we captured images of the cells at different times over a $48 \mathrm{~h}$ period. Wound width was measured at x100 magnification using a microscope. Measurements of the length of the wound were performed at random intervals, and the data were analysed by ImageJ software. This experiment was repeated three times [16].

\section{Cell invasion assay}

Transwell experiments, which are an alternative method of estimating cancer cell invasiveness, were performed in this study. Briefly, cell invasion was measured using Matrigel-coated Transwell cell culture chambers. Cells in the logarithmic phase were starved in serum-free F12k medium for $24 \mathrm{~h}$, after which they were digested by $0.25 \%$ EDTA-trypsin. The cell suspension was then treated with serum-free F12k medium, during which the density of the suspension was adjusted to $2 \times 10^{5} / \mathrm{mL}$. Approximately $200 \mu \mathrm{L}$ of the cell suspension was added to the upper portion of the Transwell insert (Corning Costar) , and F12k medium containing $10 \%$ FBS was added to the lower portion of the inset at a concentration of $600 \mu \mathrm{L} /$ well. The cells were then cultured for $24 \mathrm{~h}$ at $37^{\circ} \mathrm{C}$ with $5 \% \mathrm{CO}$. Three duplicate systems were used for each group. After $24 \mathrm{~h}$, the chamber was dislodged, fixed with methanol for $20 \mathrm{~min}$, and then dried at room temperature. The chamber was then dyed by crystal violet for $20 \mathrm{~min}$. The cells that had were unable to pass through 


\section{Cellular Physiology Cell Physiol Biochem 2018;49:335-348

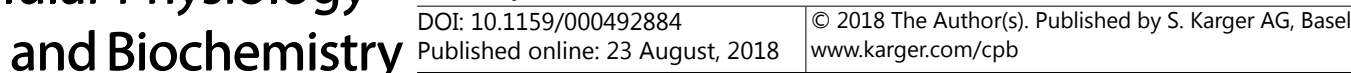

the upper portion of the chamber were removed with a wet cotton swab, and then the chamber was placed under an inverted microscope so that the remaining cells could be counted. The images were analysed by Image software $[16,17]$.

\section{Real-time PCR}

RNA was isolated from cells using Trizol reagent (TaKaRa) . One microgram of RNA per sample was reverse transcribed in a final mixture of $5 \times$ primer script buffer, primer script enzymes, oligo dT primers and reverse transcriptase (TaKaRa) . Reverse transcription was performed at $37^{\circ} \mathrm{C}$ for $15 \mathrm{~min}, 84^{\circ} \mathrm{C}$ for $5 \mathrm{~s}$ and $4{ }^{\circ} \mathrm{C}$ for the appropriate amount of time.

mRNA expression was quantified using a SYBR Green System, as previously described [18]. Expression levels of the autophagy-related and apoptosis-related genes were normalized using the $\beta$-actin gene. Each experiment was repeated three times and was performed independently in duplicate. mRNA expression up-regulation or down-regulation was quantified by the cycle threshold (CT) method, and SPSS version 11.5 (Mann-Whitney U test) was used to calculate the statistical significance of the differences in the mRNA expression levels of various genes among different samples. $\mathrm{P}<0.05$ was considered significant [19].

Western blot analysis

Twenty micrograms of protein per sample was separated on a 12\% SDS gel, after which semi-dry western blotting was performed. The membranes were subsequently blocked with 5\% BSA in TBST (50 mM Tris-HCl (pH 7.5) and $150 \mathrm{mM} \mathrm{NaCl}$ containing 0.05\% Tween 20). The blots were incubated with primary antibodies against $\beta$-actin(1:1000, antirabbit, HuaBio), LC3B (1:1000, antirabbit, Bioworld), Beclin1 (1:1000, antimouse, Bioworld), SQSTM1/P62 (1:1000, antirabbit, CST), Bcl2 (1:1000, antirabbit, CST), NF$\kappa \mathrm{B}(1: 1000$, antirabbit, abcam), caspase3 (1:1000, antirabbit, CST), Bax (1:1000, antirabbit, Bioworld), Akt (1:1000, antirabbit, CST), p-Akt (1:1000, antirabbit, CST), PTEN (1:1000, antirabbit, HuaBio) and FOXO3 (1:1000, antirabbit, Bioworld) overnight at $4{ }^{\circ} \mathrm{C}$. The membranes were incubated with HRP-conjugated secondary antibodies (1:10000 dilution, CST) for approximately $1 \mathrm{~h}$ at room temperature. Antibody staining was subsequently visualized by chemiluminescence $[6,18-20]$.

\section{Detection of mRFP-GFP-LC3}

AGS cells grown in media with different $\mathrm{pH}$ values were seeded on gelatinized coverslips and infected with mRFP-GFP-LC3 adenoviruses. The medium was replaced with fresh medium after $24 \mathrm{~h}$. Some cells were fixed with $4 \%$ paraformaldehyde for mRFP-GFP observation. The fixed cells were treated with 5\% BSA for 30 min and stained with DAPI for 6 min, after which the coverslips were air-dried and mounted in Gel Tol. mRFP-GFP-LC3 fluorescence was observed under a fluorescence microscope, as previously described $[6,21,22]$. We counted the numbers of mRFP-GFP-LC3 punctae in three independent visual fields.

\section{Statistical analysis}

All the results for our study are presented as the mean \pm SD. ANOVA was used to assess any differences among the four groups, and all statistical analyses were performed with SPSS version 15.0 statistical software. Differences were considered statistically significant at $\mathrm{P}<0.05$.

\section{Results}

FOXO3 overexpression and an acidic microenvironment effectively suppressed AGS cell growth, migration and invasion.

Previous studies have shown that acidic microenvironments drive tumour growth, invasion and autophagy [23]. However, FOX03 suppresses cancer invasion and growth but induces autophagy. To determine whether FOXO3 and an acidic microenvironment inhibited AGS cell growth and proliferation in vitro, we treated the cells with media with different $\mathrm{pHs}$ (pH 6.0 and 7.4) and FOX03-expressing plasmids or negative control plasmids. Thus, the following four different groups of cells were utilized in this study: a group of cells transfected with FOX03-expressing plasmids and treated with medium with a $\mathrm{pH}$ of 6.0, a group of cells transfected with negative control plasmids and treated with medium with a pH of 6.0, a group 


\section{Cellular Physiology Cell Physiol Biochem 2018;49:335-348 \begin{tabular}{l|l|l} 
DOI: 10.1159/000492884 & $\begin{array}{l}\text { O 2018 The Author(s). Published by S. Karger AG, Basel } \\
\text { www.karger.com/cpb }\end{array}$
\end{tabular}

of cells transfected with FOXO3-expressing plasmids and treated with medium with a pH of 7.4 and a group of cells transfected with negative control plasmids and treated with medium with a pH of 7.4. CCK8 assay was conducted to measure cell viability. As shown in Fig. 1, the results indicated that FOXO3 and low pH treatment greatly suppressed AGS cell growth $\mathrm{P}<$ 0.001). However, cells treated with only FOXO3 plasmids displayed barely detectable signs of toxicity, suggesting that this treatment had slight cytostatic effects $(\mathrm{P}<0.001)$. In addition, cell viability was slightly increased in the group of AGS cells treated with only medium with a low $\mathrm{pH}$ ( $\mathrm{pH}$ 6.0). These findings led us to conclude that FOXO3 and low-pH medium can effectively reduce AGS cell viability.

To evaluate the effect of FOXO3 on AGS cell migration and invasion in an acidic microenvironment, we performed transwell and cell wound scrape assays. We found that FOXO3 overexpression and a low $\mathrm{pH}$ led to dramatic decreases in cell migration and invasion in the group transfected with FOXO3-expressing plasmids and treated with media with a $\mathrm{pH}$ of 6.0 compared with the other groups $(\mathrm{P}<0.001$, Fig. 2A-D). However, cell migration and invasion were slightly enhanced in the group of cells treated with only FOXO3-overexpressing plasmids ( $\mathrm{P}<0.001$, Fig. 2A-D) and moderately enhanced in the group of cells treated with only medium with a low $\mathrm{pH}$. Based on these results, we concluded that the combination of FOXO3 overexpression and a low $\mathrm{pH}$ play an important role in inhibiting AGS cell migration and invasion.

To study AGS cell migration and invasion further, we assessed the expression of MMP2, MMP-9 and PTEN, proteins that play functional roles in cell migration and invasion. As shown in Fig. 2E-F, western blotting revealed that enhancing FOXO3 expression and a low pH markedly decreased MMP-2 and MMP-9 production, while significantly increased PTEN (P< $0.001)$. However, treatment with only a low $\mathrm{pH}$ slightly improved cell migration and invasion ability $(\mathrm{P}<0.001)$. In addition, FOXO3 overexpression increased cell migration and invasion ability $(\mathrm{P}<0.001)$. These results were consistent with those of the transwell and cell wound scrape experiments.

FOXO3 overexpression and an acidic microenvironment increase autophagy in AGS cells.

Previous studies have shown that acidic environments and FOXO3 transcription factors can induce autophagy in cancer cells; however, few studies have investigated whether autophagy occurs in AGS cells exposed to an acidic microenvironment and transfected with plasmids overexpressing FOXO3. To determine whether increases in FOXO3 expression and low-pH exposure enhances the expression of autophagic genes, we performed real-time quantitative RT-PCR analysis to detect LC3, Beclin1, P62, Atg5 and PTEN expression. The results showed that the basal mRNA expression levels of LC3, Beclin1, Atg5 and PTEN were

Fig. 1. FOXO3-overexpressing plasmids and low-pH microenvironment treatment had dramatic cytotoxic effects on AGS cells. AGS cells were cultured under two different $\mathrm{pHs}(\mathrm{pH} 6.0$ and $\mathrm{pH}$ 7.4) and with specific FOXO3 plasmids (positive or negative). Cell viability was analysed by CCK8 assay. Values are expressed as the mean \pm SD of four independent experiments $(n=4$, $\mathrm{F}=320.4, \mathrm{P}<0.001$ ).

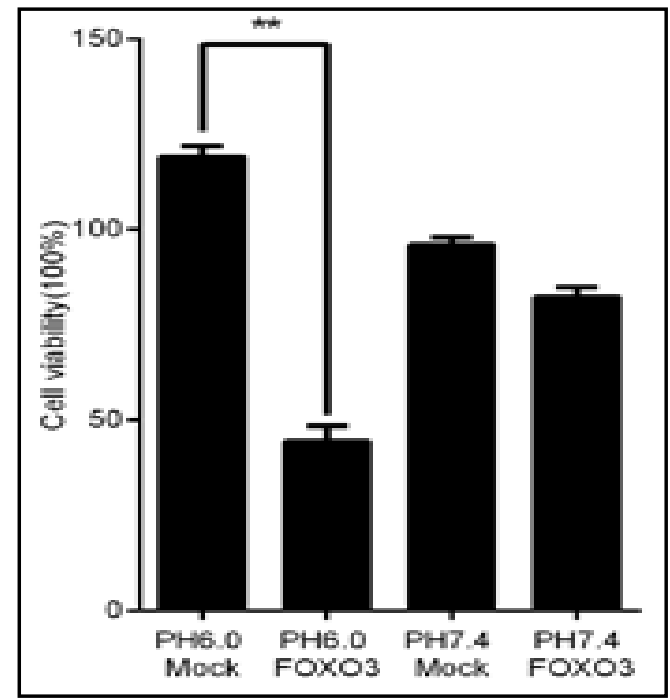




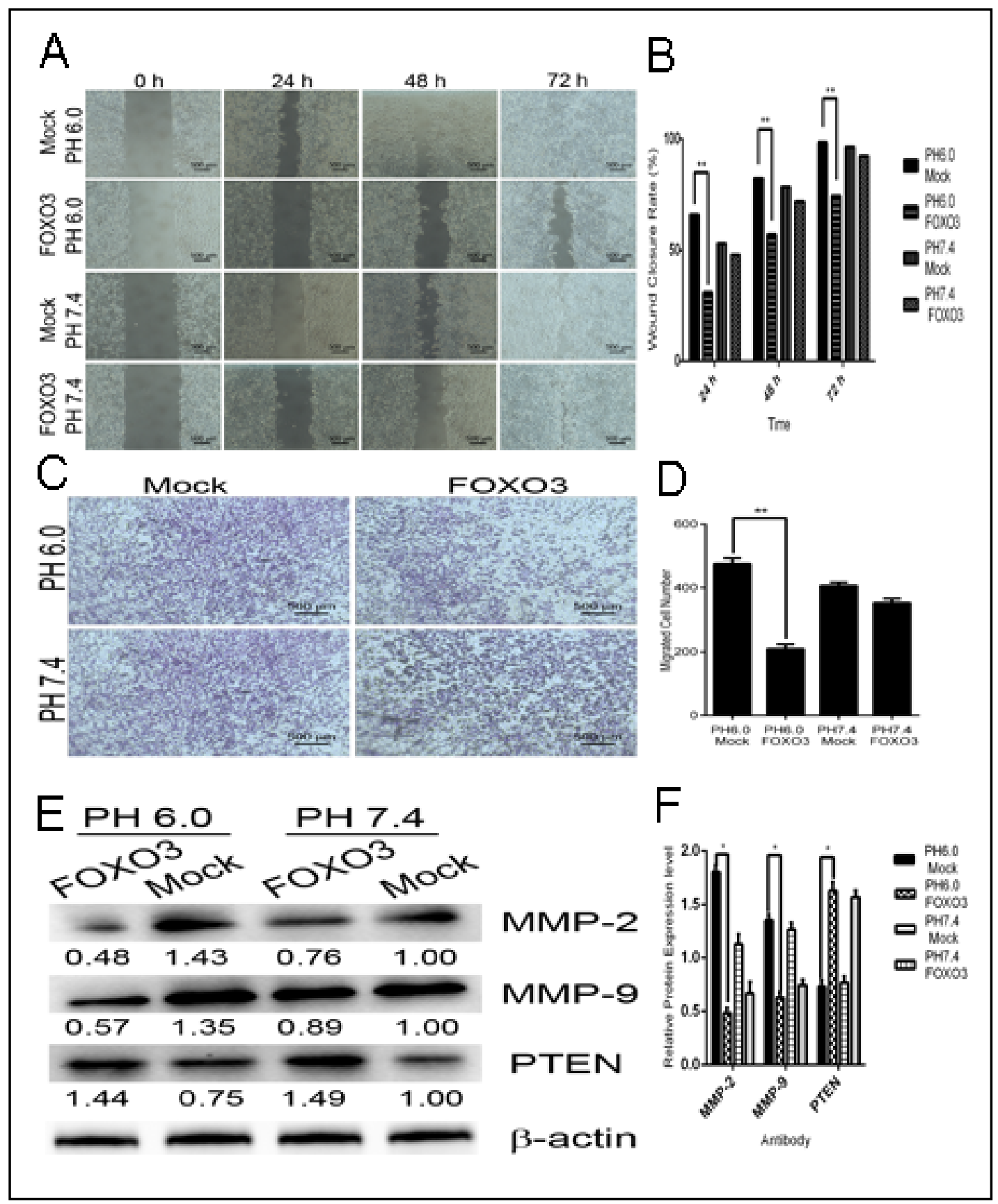

Fig. 2. FOXO3-overexpressing plasmids and low-pH microenvironment treatment inhibit AGS migration and invasion. A-B. Cell migration ability, as detected by wound-scrape assay. The cells were damaged by mechanical scraping. Representative images of cell migration in the wound scrape model at 0, 24 and $48 \mathrm{~h}$ post-wound induction are shown (magnification, $\mathrm{x} 40$ ). The migrating cell area is expressed as the mean \pm SD of 3 independent experiments $(n=4, F=220.1, P<0.001)$. C-D. Cell invasion ability, as detected by Transwell assay. Representative images of the invading cells. (Magnification, $\mathrm{x} 40$ ). The number of invading cells is expressed as the mean \pm SD of 3 independent experiments $(n=4, F=214.3, P<0.001)$. E-F. Western blot analysis was performed on the lysates of AGS cells treated under four different conditions to detect the protein expression levels of MMP-2 ( $\mathrm{n}=4, \mathrm{~F}=183.3, \mathrm{P}<0.001)$, MMP-9 $(\mathrm{n}=4, \mathrm{~F}=165.7, \mathrm{P}<0.001)$ and PTEN $(\mathrm{n}=4, \mathrm{~F}=151.1, \mathrm{P}<0.001)$, which participate in migration and invasion. Quantitative analysis of the levels of these three proteins was also performed (bottom panel). Values are expressed as the mean \pm SD of three independent experiments. Statistical comparisons were performed with one-way ANOVA test.

\section{KARGER}


low in AGS cells. However, incubation with FOXO3-overexpressing plasmids and media with a low $\mathrm{pH}$ resulted in significant increases in the mRNA expression levels of the above proteins compared with control cells. Additionally, AGS cells treated with only FOXO3-overexpressing plasmids or a low-pH environment showed no clear increases in the mRNA expression levels of the above proteins compared with control cells ( $\mathrm{P}<0.05$, Fig. 3). P62 mRNA expression levels were greatly decreased after treatment with FOXO3-overexpressing plasmid- and low pH-treated cells compared with control cells. However, cells treated with only FOXO3overexpressing plasmids or a low-pH environment displayed no significant reduction in P62 mRNA expression levels compared with control cells $(\mathrm{P}<0.05$, Fig. 3 ).

In summary, these results showed that AGS cells treated with FOXO3-overexpressing plasmids and low $\mathrm{pH}$ displayed enhanced autophagy compared with cells treated with only FOXO3-overexpressing plasmids or a low-pH environment.

In subsequent studies, we used western blotting to test the expression of the following essential protein markers of autophagy: LC3I and LC3II, Beclin1, FOXO3, Akt, p-Akt and P62. As shown in Fig. 4, LC3I and FOXO3 expression levels were significantly elevated in the FOXO3-overexpressing plasmid- and low $\mathrm{pH}$-treated group compared with the other groups $(\mathrm{P}<0.05$, Fig. 4). Increased LC3II expression commonly serves as an indicator of elevated autophagy levels in mammalian cell models, and the LC3I/LC3II, ratio was significantly increased in the FOXO3-overexpressing plasmid- and low pH-treated group. However, the group treated with only FOXO3-overexpressing plasmids did not display increases in the levels of these protein markers of autophagy but expressed the proteins at higher levels than the remaining two groups. Consistent with these results, the level of the autophagy

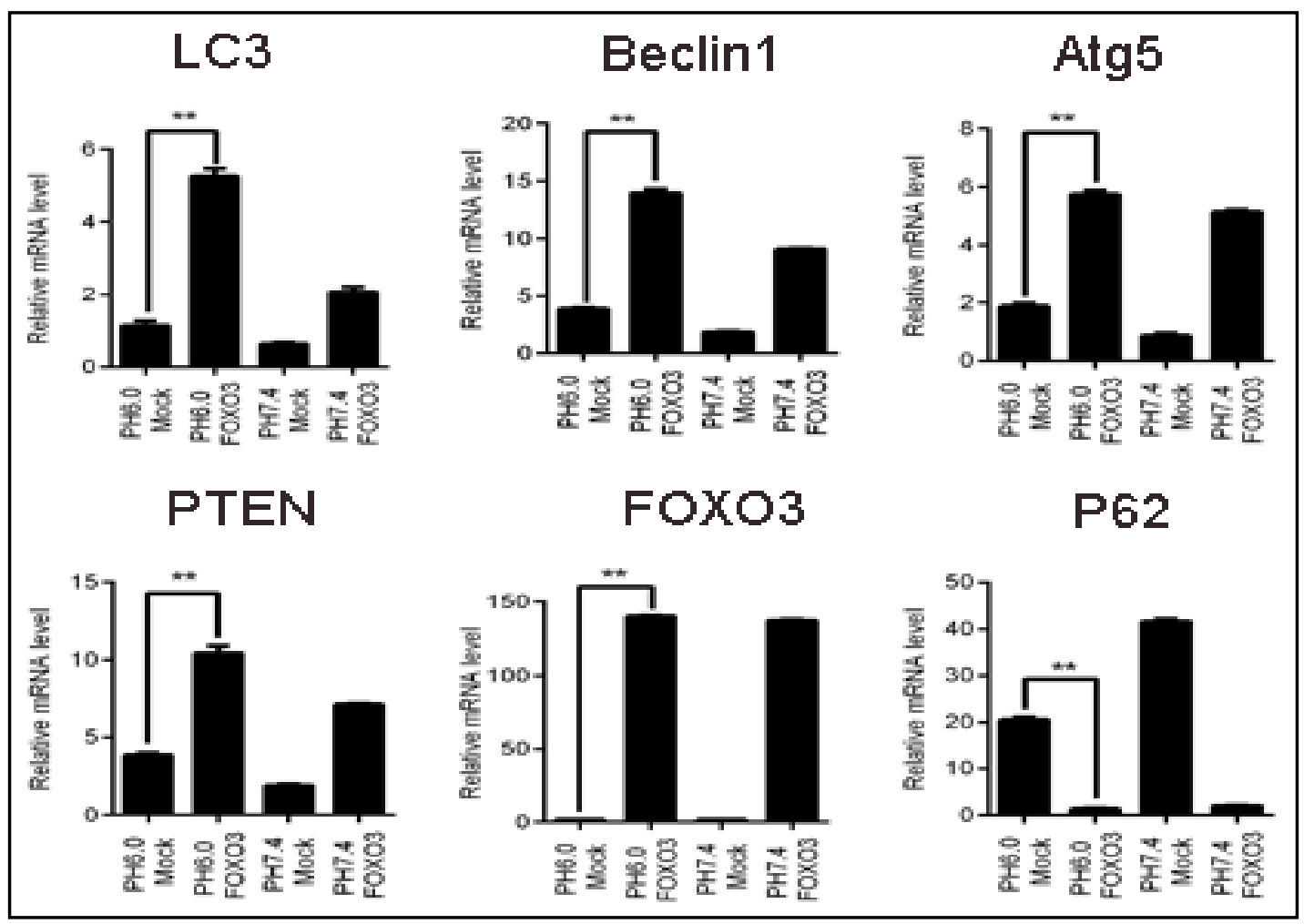

Fig. 3. Real-time quantitative RT-PCR analysis of the expression of autophagy-related genes in AGS cells treated with FOXO3-overexpressing plasmids and a low-pH microenvironment. The expression of autophagyrelated genes, such as LC3 ( $\mathrm{n}=4, \mathrm{~F}=659.2, \mathrm{P}<0.001)$, Beclin1 ( $\mathrm{n}=4, \mathrm{~F}=1931, \mathrm{P}<0.001), \operatorname{Atg} 5(\mathrm{n}=4, \mathrm{~F}=288.3, \mathrm{P}$ $<0.001)$, PTEN ( $\mathrm{n}=4, \mathrm{~F}=757.1, \mathrm{P}<0.001)$, FOX03 ( $\mathrm{n}=4, \mathrm{~F}=119452, \mathrm{P}<0.001)$ and $\mathrm{P} 62(\mathrm{n}=4, \mathrm{~F}=6573, \mathrm{P}<0.001)$, was tested by RT-PCR. Values are expressed as the mean \pm SD of three independent experiments. Statistical analysis was performed with SPSS followed by one-way ANOVA test.

\section{KARGER}




\section{$\begin{array}{lll}\text { Cellular Physiology } & \text { Cell Physiol Biochem 2018;49:335-348 } \\ \text { DOI: 10.1159/000492884 } & \text { O 2018 The Author(s). Published by S. Karger AG, Basel }\end{array}$

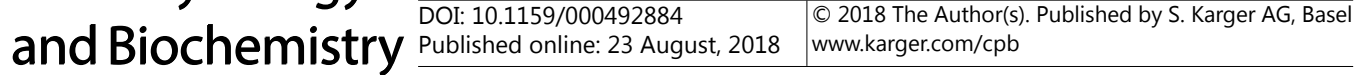 \\ Gao et al.: FOXO3 Inhibits AGS Cell Growth via Autophagy}

substrate P62 was significantly reduced in the FOX03-overexpressing plasmid- and low $\mathrm{pH}$ treated group compared with the other groups $(\mathrm{P}<0.05)$. AGS cells treated with only FOXO3overexpressing plasmids displayed no clear decreases in P62 expression but expressed the protein at a lower level than the other two groups. These results suggested that FOXO3overexpressing plasmids and low-pH treatment enhance autophagy in AGS cells.

The PI3K/Akt axis has been identified as a regulator of normal metabolic activity and a negative regulator of autophagic activity [20,24]. To investigate whether the activity of this pathway changes in FOXO3-overexpressing plasmid- and low pH-treated AGS cells, we analysed the expression levels of various phospho-proteins in the cells. We noted significant decreases $(\mathrm{P}<0.05)$ in phospho-Akt levels (Fig. 4) in cells treated with low-pH medium and FOXO3-overexpressing plasmids compared with control cells; however, Akt expression changed slightly in the treated group of cells compared with the control group cells $(\mathrm{P}<0.05$, Fig. 4). These results suggested that these phospho-proteins are involved in mediating the effects of FOXO3 overexpression and low pH on autophagy in AGS cells.

Based on the above results, we concluded that FOXO3 overexpression and a low $\mathrm{pH}$ induced autophagy through the PI3K/Akt pathway, of which FOXO3 appears to be a downstream effector, as shown in previous studies [25]. Previous studies also suggested that decreased PI3K/Akt signalling activates autophagy through not only mTOR but also more slower transcription-dependent processes involving FOXO3. We found that acidic microenvironment-induced stress and FOXO3 overexpression induce autophagy; however,

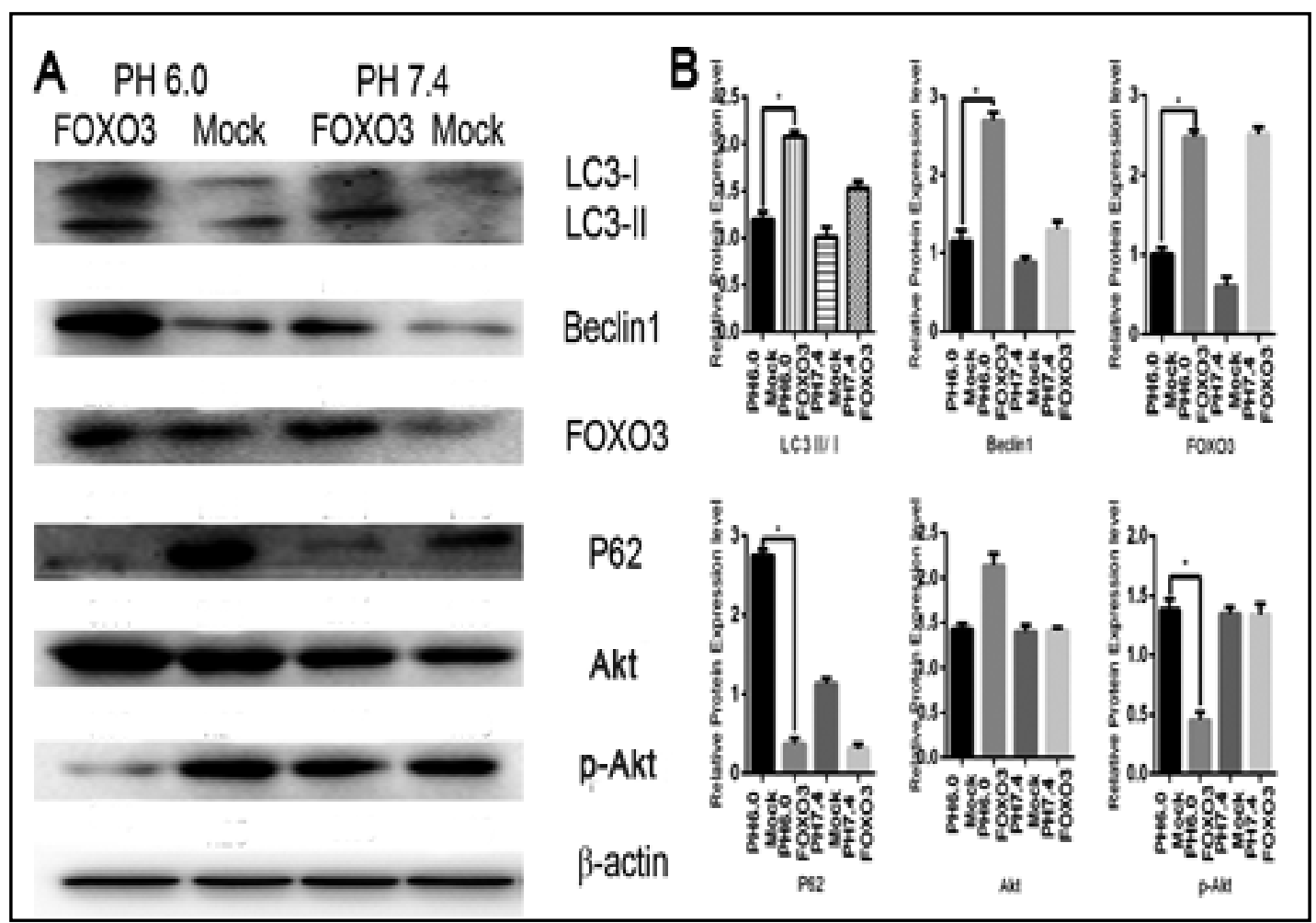

Fig. 4. A-B. FOXO3 overexpression and a low-pH microenvironment induced autophagy in AGS cells. The expression of LC3 II /LC3 I ( $\mathrm{n}=4, \mathrm{~F}=49.9, \mathrm{P}<0.05)$ and other autophagy-related proteins, such as Beclin1 $(\mathrm{n}=4, \mathrm{~F}=137.5, \mathrm{P}<0.05), \mathrm{FOX03}(\mathrm{n}=4, \mathrm{~F}=202.9, \mathrm{P}<0.001)$ and $\mathrm{P} 62(\mathrm{n}=4, \mathrm{~F}=240.2, \mathrm{P}<0.001)$, was analysed by western blotting. The expression of proteins related to the PI3K/Akt pathway, namely, Akt ( $n=4, F=19.7$, $\mathrm{P}<0.05)$ and $\mathrm{p}-\mathrm{Akt}(\mathrm{n}=4, \mathrm{~F}=184.6, \mathrm{P}<0.001)$, were detected by western blotting. Quantitative analysis of the expression of these seven protein levels was also performed (bottom panel). Values are expressed as the mean \pm SD of three independent experiments. Statistical comparisons were performed with one-way ANOVA test. 
the detailed mechanisms underlying this phenomenon remain unclear.

To confirm whether or not FOXO3 overexpression and low pH treatment induces autophagic flux, we analysed AGS cells transiently transfected with mRFP-GFP-LC3B adenoviruses under a laser scanning confocal microscope. An emerging system involving the tandem fusion of LC3 to acid-resistant mCherry (such as RFP) and acid-sensitive GFP has been applied for the analysis of autophagosome-mediated dynamic changes in proteins and protein degradation to monitor autophagic flux, as GFP fluorescence is quenched in low $\mathrm{pH}$ compartments. A dynamic switch from yellow to red fluorescence reflects functional autophagic flux. In this system, LC3 is fused to both GFP and RFP to form an RFP-GFPLC3 vector. As shown in Fig. 5, cells incubated with FOXO3-overexpressing plasmids and under low-pH conditions for $24 \mathrm{~h}$ displayed markedly increased numbers of yellow puncta

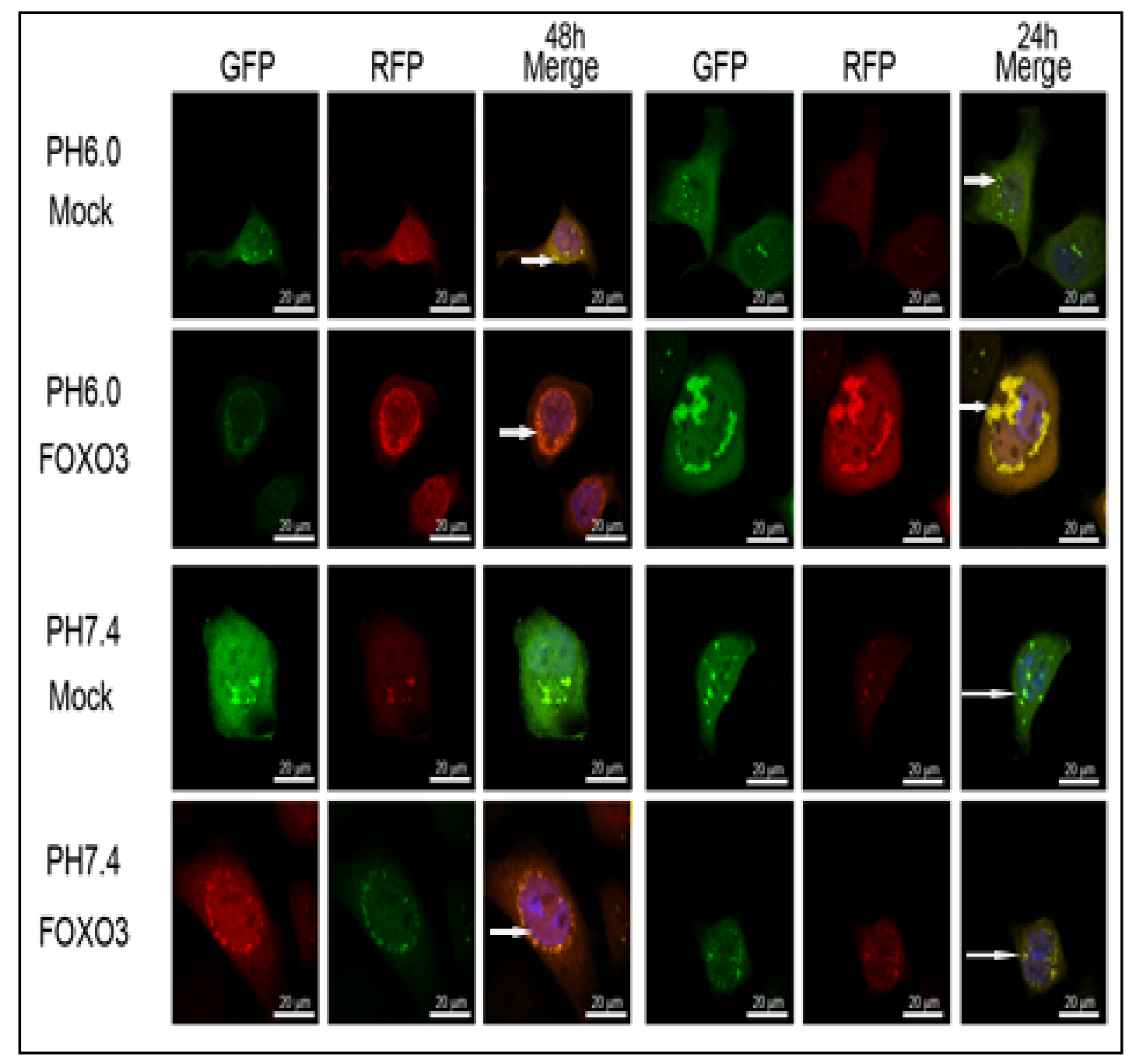

Fig. 5. FOXO3 overexpression and acidic stress promote autophagosome formation and increase autophagic flux. AGS cells incubated under four different conditions were transfected with the RFP-GFP-LC3 adenoviral vector. After 24h $(\mathrm{n}=4, \mathrm{~F}=140.3, \mathrm{P}<0.001)$ or $48 \mathrm{~h}(\mathrm{n}=4, \mathrm{~F}=1190, \mathrm{P}<0.001)$ of transfection, the AGS cells were observed under a confocal microscope. Autophagic flux was measured by counting the cells with GFP $+/ \mathrm{mRFP}+$ (yellow) and GFP - /mRFP + (red) LC3 puncta. Yellow and red punctas are indicated by white arrows. Nuclear staining is apparently visible in the merge panel which colored blue. Data are representative of three independent experiments. All the groups were compared with the control group by one-way ANOVA test with SPSS.

\section{KARGER}


stably expressing mRFP-GFP-LC3B compared with other cells $(\mathrm{P}<0.05)$, suggesting that FOXO3 overexpression and acidic stress promote autophagosome formation. After 48 $\mathrm{h}$, the yellow fluorescence switched to red fluorescence in the group of cells treated with FOXO3-overexpressing plasmids and low-pH medium ( $\mathrm{P}<0.05$, Fig. 5), implying that FOXO3 overexpression and acidic stress increase autophagic flux. Taken together, our data suggest that FOXO3 overexpression and a low $\mathrm{pH}$ have dynamic effects on autophagic flux and induce late increases in autophagic flux.

FOXO3 overexpression and acidic stress induce apoptotic cell death by enhancing autophagy induction in AGS cells.

To determine whether apoptosis is mediated by autophagy induction in FOX03overexpressing plasmid- and low pH-treated AGS cells, we first measured the expression of apoptosis-related mRNAs (such as Bcl2 and Bax) in cells treated with FOXO3-overexpressing plasmids and low-pH medium by RT-PCR analyses. As shown in Fig. 6, FOXO3 overexpression and acid-induced stress increased the $\mathrm{Bax} / \mathrm{Bcl} 2$ ratio, suggesting that FOXO3 overexpression and acidic stress induce apoptosis.

Apoptosis occurs via extrinsic, intrinsic and ER stress-mediated processes. All three apoptotic processes eventually rely on the enzymatic activity of caspase 3 and result in the enzymatic degradation of the downstream protein. As shown in Fig. 7, AGS cells treated with FOXO3-overexpressing plasmids and low-pH medium displayed statistically significant increases in Bax and cleaved caspase 3 expression compared with cells treated with FOXO3overexpressing plasmids alone $(\mathrm{P}<0.05)$. The abovementioned apoptosis-related proteins are all pro-apoptotic proteins. These results suggest that FOXO3 overexpression and acidic stress promote apoptosis progression. In addition, we also analysed the expression of $\mathrm{Bcl} 2$ and NF-кB, which are anti-apoptotic proteins. As shown in Fig. 7, we noted that the levels of these proteins were clearly reduced in the FOXO3-overexpressing plasmid- and low $\mathrm{pH}$ treated group. However, cells treated with FOXO3-overexpressing plasmids alone exhibited relatively weak Bcl2 and NF- $\kappa$ B expression $(\mathrm{P}<0.05)$.

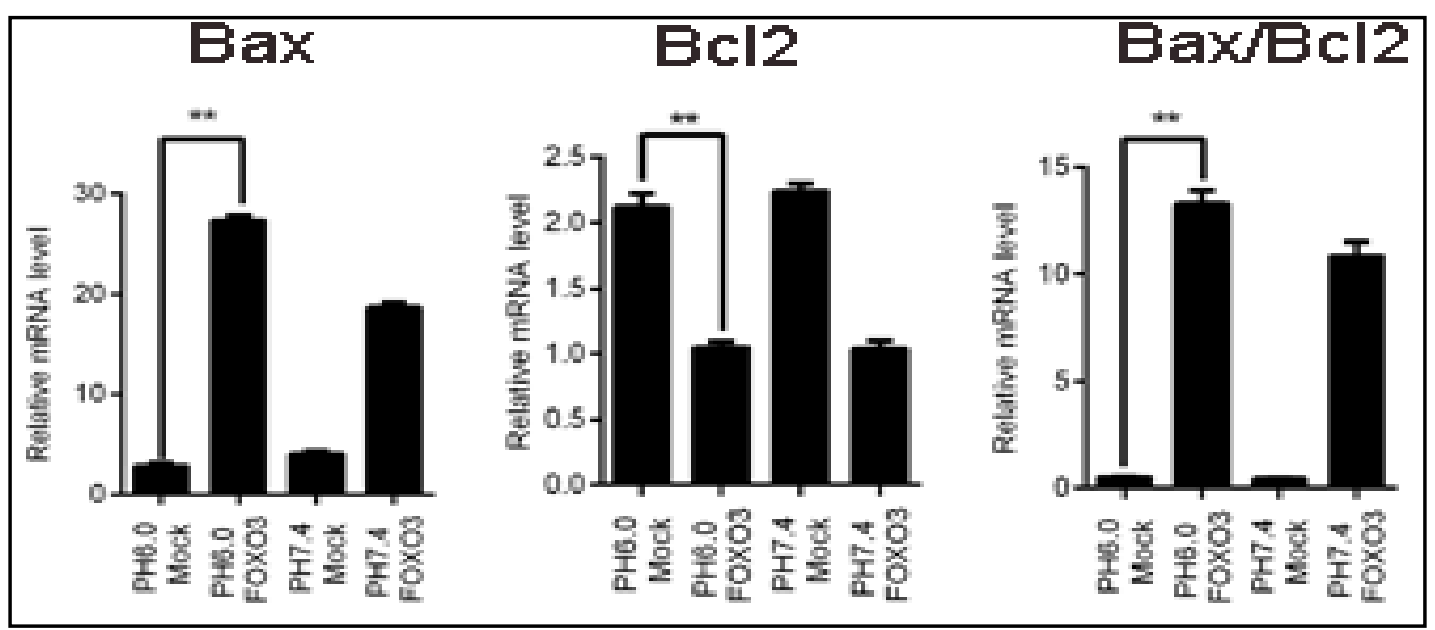

Fig. 6. FOXO3 overexpression and acidic stress induce apoptotic cell death, as determined by real-time quantitative RT-PCR. Real-time quantitative RT-PCR showed that the expression levels of apoptosis-related proteins changed in AGS cells after treatment with FOXO3-overexpressing plasmids and low-pH medium. The expression of apoptosis-related genes, such as Bcl2 ( $\mathrm{n}=4, \mathrm{~F}=246.2, \mathrm{P}<0.001)$ and $\mathrm{Bax}(\mathrm{n}=4, \mathrm{~F}=4122$, $\mathrm{P}<0.001)$, was tested, and the $\mathrm{Bax} / \mathrm{Bcl} 2(\mathrm{n}=4, \mathrm{~F}=580.6, \mathrm{P}<0.001)$ ratio was calculated, as shown. The data are expressed as the mean \pm SD. Statistically significant versus control. 


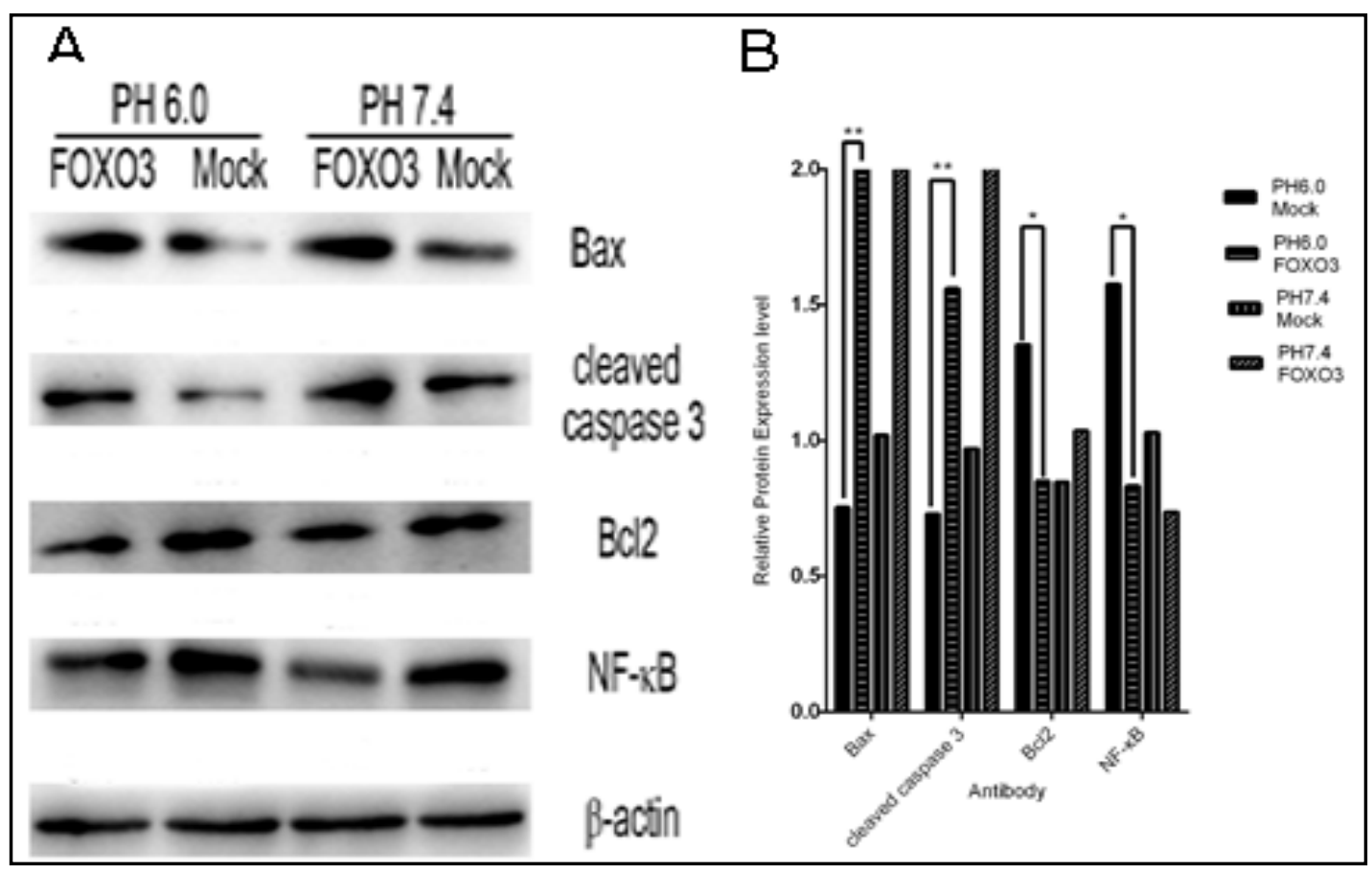

Fig. 7. Western blot analysis of the expression of apoptosis-related proteins in AGS cells treated with FOXO3overexpressing plasmids and a low-pH microenvironment. Western blot analyses were performed on AGS cells treated with FOXO3 overexpression and low $\mathrm{pH}$ to detect the protein levels of $\mathrm{Bax}(\mathrm{n}=4, \mathrm{~F}=241.3$, $\mathrm{P}<0.001)$, caspase3 ( $\mathrm{n}=4, \mathrm{~F}=250.1, \mathrm{P}<0.001), \mathrm{Bcl} 2(\mathrm{n}=4, \mathrm{~F}=35.3, \mathrm{P}<0.05)$ and $\mathrm{NF}-\kappa \mathrm{B}(\mathrm{n}=4, \mathrm{~F}=145.1, \mathrm{P}<0.001)$, which are markers of apoptosis. Quantitative analysis of the expression levels of these proteins was also performed (bottom panel). Values are expressed as the mean \pm SD of three independent experiments. Statistical analysis was performed with one-way ANOVA test.

\section{Discussion}

Previous studies have shown that tumour cells exposed to acidic conditions undergo metabolic changes and that the up-regulation of glycolysis in these cells results in the extracellular accumulation of metabolic acids [26]. Such acidic microenvironments induce malignant progression [6]. It has been shown that acidic $\mathrm{pH}$ promotes local invasive growth and metastasis. In addition, autophagy can also be induced by extracellular acidosis and appears to serve as an adaptation enabling cells to survive in acidic microenvironments [27]. Autophagy is a cellular catabolic process that induces the lysosomal degradation and recycling of dysfunctional proteins or organelles, processes that are considered vital to the survival of cancer cells exposed to acidic stress [28]. We have determined that autophagy plays a complex role in tumour progression. In the early stage of tumour development, autophagy acts as a tumour inhibitor by scavenging mitochondrial proteins whose folding was disrupted by reactive oxygen species (ROS) [29, 30]. However, in the setting of metabolic stresses, such as acidic conditions, autophagy plays a role in the survival of tumour cells by enabling them to resist the metabolic stress caused by inadequate nutrition and excessive cell proliferation [31].

Our study indicated that autophagy levels increased in FOXO3 protein overexpression and that FOXO3 was expressed at higher levels in low-pH ( $\mathrm{pH} 6.0$ ) conditions than in normal ( $\mathrm{pH}$ 7.4) conditions.Based on these findings, we concluded that FOXO3, which belongs to a family of transcription proteins, is involved in the regulation of autophagy. Increases in FOXO3 expression induce autophagy [12]. Previous studies have shown that FOXO3 is closely related to tumourigenesis and is considered a tumour suppressor [13]. Current studies have also illustrated that FOXO3 is involved in autophagy and may serve as a new

\section{KARGER}


target for anti-tumour therapies [32,33]. The results of these studies are consistent with our results, which showed that FOXO3 plays a protective role in gastric tumour cells in abnormal microenvironments, such as acidic microenvironments. Our data show that AGS cells with FOXO3 overexpression that were exposed to an acidic microenvironment (pH 6.0) displayed consistent increases in the expression of autophagy marker proteins, such as LC3 and Beclin1, and decreases in the expression of P62 compared with control cells. However, AGS cells treated with FOXO3-overexpressing plasmids did not show increases in LC3 and Beclin1 expression or decreases in P62 expression. These results imply that acidic stress enhances FOX03-induced autophagy; however, the mechanism by which this occurs is not clear.

The relationship between autophagy and apoptosis is very complicated. Previous scholars proposed that autophagy activates apoptosis. There has been great progress in the understanding of autophagy since 2003; however, the relationship between autophagy and apoptosis remains controversial [34].

In conclusion, our study revealed that the FOXO3 protein plays an important role in autophagy in GC cells exposed to an acidic microenvironment. Thus, FOXO3 may promote autophagy in gastric tumours exposed to an acidic microenvironment. Recent studies have shown that the PI3K/Akt axis acts as a negative regulator of autophagy and apoptosis [20,24]. The protein kinase Akt can be activated to phospho-Akt, which enhances the transcriptional activity of NF- $\kappa$ B to up-regulate the expression of the anti-apoptotic protein Bcl2. Based on the results of our study, we propose that PI3K/Akt signalling activates autophagy through a transcription-dependent mechanism involving FOXO3 rather than mTOR. Briefly, FOXO3 up-regulation and acidic stress play important roles in inducing apoptosis and autophagy. Autophagy is a lysosome-dependent cellular degradative process that may lead to cell death in extreme cases and appears to be a special mechanism through which tumours are suppressed. FOXO3 overexpression dramatically increases autophagy levels in AGS cells and thus perhaps contributes to the tumour suppressor functions of FOXO3 [9]. These findings not only suggest that FOXO3 and acidic stress can serve as novel autophagy enhancers but also indicate that they are excellent tools for determining how autophagy and apoptosis are regulated by PI3K/Akt signalling pathway activation in AGS cells.

Thus, the results described herein indicate that autophagy induces apoptosis in AGS cells under acidic stress. Additionally, our data show that AGS cells survive in an acidic microenvironment by activating adaptive autophagy. A more thorough understanding of the molecular mechanisms by which FOXO3 and acidic stress induce autophagy and apoptosis will facilitate the development of candidate compounds that may be used to treat GC. Given the basal role of autophagy in tumourigenesis and the fact that FOXO3 is highly expressed in GC, additional studies are needed to assess the role of FOXO3 overexpression and low-pH microenvironments in autophagy in GC development.

\section{Conclusion}

Taken together, our results show that FOXO3 inhibits AGS cell growth by promoting autophagy in an acidic microenvironment. The exact mechanisms by which FOXO3 overexpression and low-pH microenvironments promote autophagy to inhibit AGS cell growth still need more research; however, our study indicates that FOXO3 and low-pH microenvironments may serve as new and accurate prognostic factors in GC in the future.

Based on our research, we suggest that expression of FOXO3 will help to recognize patients that benefit from PI3K/Akt inhibition and/or FOXO3 activation treatments, allowing the develop of new therapeutic strategies that can creat a new situation for the treatment of GC patients. 


\section{Cellular Physiology Cell Physiol Biochem 2018;49:335-348 \begin{tabular}{ll|l} 
DOI: 10.1159/000492884 & $\begin{array}{l}\text { O 2018 The Author(s). Published by S. Karger AG, Basel } \\
\text { www.karger.com/cpb }\end{array}$
\end{tabular}}

Gao et al.: FOXO3 Inhibits AGS Cell Growth via Autophagy

\section{Acknowledgements}

The authors gratefully acknowledge the financial support from the Natural Science Foundation of China $(81472338,81602068)$ and Shandong Province key research and development programs (2016GSF201138). Yuan Gao and Weiwei Qi analyzed data and wrote the manuscript. Libin Sun assisted in editing the manuscript. Jing lv contributed to the design of the study. Wensheng Qiu and Shihai Liu are the corresponding authors of the paper. All authors read and approved the final manuscript.

\section{Disclosure Statement}

All authors declare no conflicts of interest.

\section{References}

1 Sandri M: FOXOphagy path to inducing stress resistance and cell survival. Nat Cell Biol 2012;14:786-788.

-2 Choi PR, Kang YJ, Sung B, Kim JH, Moon HR, Chung HY, Kim SE, Park MI, Park SJ, Kim ND: MHY218-induced apoptotic cell death is enhanced by the inhibition of autophagy in AGS human gastric cancer cells. Int J Oncol 2015;47:563-572.

-3 Yu M, Gou WF, Zhao S, Xiao LJ, Mao XY, Xing YN, Takahashi H, Takano Y, Zheng HC: Beclin 1 expression is an independent prognostic factor for gastric carcinomas. Tumour Biol 2013;34:1071-1083.

4 Xu T, Su H, Ganapathy S, Yuan ZM: Modulation of autophagic activity by extracellular pH. Autophagy 2011;7:1316-1322.

5 Yang X, Yu DD, Yan F, Jing YY, Han ZP, Sun K, Liang L, Hou J, Wei LX: The role of autophagy induced by tumor microenvironment in different cells and stages of cancer. Cell Biosci 2015;5:14.

6 Zhang Z, Lai Q Li Y, Xu C, Tang X, Ci J, Sun S, Xu B, Li Y: Acidic pH environment induces autophagy in osteoblasts. Sci Rep 2017;7:46161.

7 Mizushima N, Levine B, Cuervo AM, Klionsky DJ: Autophagy fights disease through cellular self-digestion. Nature 2008;451:1069-1075.

8 Zhou J, Liao W, Yang J, Ma K, Li X, Wang Y, Wang D, Wang L, Zhang Y, Yin Y, et al.: FOXO3 induces FOXO1dependent autophagy by activating the AKT1 signaling pathway. Autophagy 2014;8:1712-1723.

-9 Zhang Y, Gan B, Liu D, Paik J-h: FoxO family members in cancer. Cancer Biol Ther 2014;12:253-259.

10 Park SH, Jang KY, Kim MJ, Yoon S, Jo Y, Kwon SM, Kim KM, Kwon KS, Kim CY, Woo HG: Tumor suppressive effect of PARP1 and FOXO3A in gastric cancers and its clinical implications. Oncotarget 2015;6:4481944831.

-11 Shan Y YY, Wen Z, Wei Y, Naiqing Z, Qian L, Yuehong C, Yan W, Wei L, Yihong S, Tianshu L: FOXO3a promotes gastric cancer cell migration and invasion through the induction of cathepsin L. Oncotarget 2016;7:34773-34784.

12 Soltany-Rezaee-Rad M, Mottaghi-Dastjerdi N, Setayesh N, Roshandel G, Ebrahimifard F, Sepehrizadeh Z: Overexpression of FOXO3, MYD88, and GAPDH Identified by Suppression Subtractive Hybridization in Esophageal Cancer Is Associated with Autophagy. Gastroenterol Res Pract 2014;2014:185035-185042.

13 Yang W, Du WW, Li X, Yee AJ, Yang BB: Foxo3 activity promoted by non-coding effects of circular RNA and Foxo3 pseudogene in the inhibition of tumor growth and angiogenesis. Oncogene 2015;35:3919-3931.

14 Zhao J, Brault JJ, Schild A, Cao P, Sandri M, Schiaffino S, Lecker SH, Goldberg AL: FoxO3 coordinately activates protein degradation by the autophagic/lysosomal and proteasomal pathways in atrophying muscle cells. Cell Metab 2007;6:472-483.

15 Jing X, Xu Y, Cheng W, Guo S, Zou Y, He L: Tanshinone I induces apoptosis and pro-survival autophagy in gastric cancers. Cancer Chemother Pharmacol 2016;77:1171-1181.

16 Zhao X, Li DC, Zhu XG, Gan WJ, Li Z, Xiong F, Zhang ZX, Zhang GB, Zhang XG, Zhao H: B7-H3 overexpression in pancreatic cancer promotes tumor progression. Int J Mol Med 2013;31:283-291. 


\section{Cellular Physiology Cell Physiol Biochem 2018;49:335-348 \begin{tabular}{l|l|l} 
DOI: 10.1159/000492884 & $\begin{array}{l}\text { O 2018 The Author(s). Published by S. Karger AG, Basel } \\
\text { www.karger.com/cpb }\end{array}$
\end{tabular} \\ Gao et al.: FOXO3 Inhibits AGS Cell Growth via Autophagy}

17 Shen J, Zhao DS, Li MZ: TGF-beta1 promotes human gastric carcinoma SGC7901 cells invasion by inducing autophagy. Eur Rev Med Pharmacol Sci 2017;21:1013-1019.

-18 Karger S, Weidinger C, Krause K, Sheu SY, Aigner T, Gimm 0, Schmid KW, Dralle H, Fuhrer D: FOXO3a: a novel player in thyroid carcinogenesis? Endocr Relat Cancer 2009;16:189-199.

-19 Weidinger C, Krause K, Mueller K, Klagge A, Fuhrer D: FOXO3 Is Inhibited by Oncogenic PI3K/Akt Signaling but Can Be Reactivated by the NSAID Sulindac Sulfide. J Clin Endocrinol Metab 2011;96:1361-1371.

-20 Wojtkowiak JW, Rothberg JM, Kumar V, Schramm KJ, Haller E, Proemsey JB, Lloyd MC, Sloane BF, Gillies $\mathrm{RJ}$ : Chronic autophagy is a cellular adaptation to tumor acidic $\mathrm{pH}$ microenvironments. Cancer Res 2012;72:3938-3947.

-21 Klionsky DJ, Abdelmohsen K, Abe A, Abedin MJ, Abeliovich H, Acevedo Arozena A, Adachi H, Adams CM, Adams PD, Adeli K, et al.: Guidelines for the use and interpretation of assays for monitoring autophagy (3rd edition). Autophagy 2016;12:1-222.

22 Maejima Y, Kyoi S, Zhai P, Liu T, Li H, Ivessa A, Sciarretta S, Del Re DP, Zablocki DK, Hsu CP, et al.: Mst1 inhibits autophagy by promoting the interaction between Beclin1 and Bcl-2. Nat Med 2013;19:1478-1488.

23 Marino ML, Pellegrini P, Di Lernia G, Djavaheri-Mergny M, Brnjic S, Zhang X, Hagg M, Linder S, Fais S, Codogno P, et al.: Autophagy is a protective mechanism for human melanoma cells under acidic stress. J Biol Chem 2012;287:30664-30676.

24 Jung CH, Ro SH, Cao J, Otto NM, Kim DH: mTOR regulation of autophagy. FEBS Lett 2010;584:1287-1295.

25 Yang K, Lu Y, Xie F, Zou H, Fan X, Li B, Li W, Zhang W, Mei L, Feng SS, et al.: Cationic liposomes induce cell necrosis through lysosomal dysfunction and late-stage autophagic flux inhibition. Nanomedicine (Lond) 2016;11:3117-3137.

-26 Gatenby RA, Gillies RJ: Why do cancers have high aerobic glycolysis? Nat Rev Cancer 2004;4:891-899.

-27 Estrella V, Chen T, Lloyd M, Wojtkowiak J, Cornnell HH, Ibrahim-Hashim A, Bailey K, Balagurunathan Y, Rothberg JM, Sloane BF, et al.: Acidity generated by the tumor microenvironment drives local invasion. Cancer Res 2013;73:1524-1535.

28 Janku F, McConkey DJ, Hong DS, Kurzrock R: Autophagy as a target for anticancer therapy. Nat Rev Clin Oncol 2011;8:528-539.

29 Takamura A, Komatsu M, Hara T, Sakamoto A, Kishi C, Waguri S, Eishi Y, Hino O, Tanaka K, Mizushima N: Autophagy-deficient mice develop multiple liver tumors. Genes Dev 2011;25:795-800.

30 White E, DiPaola RS: The double-edged sword of autophagy modulation in cancer. Clin Cancer Res 2009;15:5308-5316.

31 Wang RC, Wei Y, An Z, Zou Z, Xiao G, Bhagat G, White M, Reichelt J, Levine B: Akt-mediated regulation of autophagy and tumorigenesis through Beclin 1 phosphorylation. Science 2012;338:956-959.

32 Cheong JK, Zhang F, Chua PJ, Bay BH, Thorburn A, Virshup DM: Casein kinase 1alpha-dependent feedback loop controls autophagy in RAS-driven cancers. J Clin Invest 2015;125:1401-1418.

-33 Lu Z, Yang H, Sutton MN, Yang M, Clarke CH, Liao WS, Bast RC, Jr.: ARHI (DIRAS3) induces autophagy in ovarian cancer cells by downregulating the epidermal growth factor receptor, inhibiting PI3K and Ras/ MAP signaling and activating the FOXo3a-mediated induction of Rab7. Cell Death Differ 2014;21:12751289.

-34 Xing C, Zhu B, Liu H, Yao H, Zhang L: Class I phosphatidylinositol 3-kinase inhibitor LY294002 activates autophagy and induces apoptosis through p53 pathway in gastric cancer cell line SGC7901. Acta Biochim Biophys Sin (Shanghai) 2008;40:194-201. 